\title{
26291 - ANTHROPOMETRIC VARIABLES AND LUMBAR DURAL SAC WIDTH USING ULTRASOUND
}

\author{
Cristian Arzola MD, Cristian Arzola, MD; Sharon Davies, MD; Ayman Rofaeel, \\ Jose CA Carvalho, MD PhD \\ Mount Sinai Hospital And University of Toronto, Toronto, ONTARIO, Canada
}

Anthropometric Variables and Lumbar Dural Sac Width using Ultrasound

\author{
AUTHORS: C. Arzola, S. Davies, A. Rofaeel, J. Carvalho; \\ AFFILIATION:Department of Anesthesia and Pain Management, Mount Sinai Hospital \\ and University of Toronto, Toronto, ON, Canada.
}

INTRODUCTION: It has been shown that anthropometric variables influence the CSF volume, as determined by MRI (1). This information would be important to predict spread of local anesthetics in the intrathecal space. Although MRI is unfortunately not readily available, spine ultrasound imaging is and has recently been proposed as a potential tool in the clinical setting. The purpose of this study was to investigate the correlation between anthropometric variables and the lumbar dural sac width, assessed by ultrasound, hypothesizing that the dural sac width could be an indicator of CSF volume in obstetric patients.

METHODS: After REB approval and written informed consent, 61 patients requesting labor epidurals were enrolled. Patient variables recorded were: age, height and weight. Body mass index $(\mathrm{BMI}=$ weight/height 2 in $\mathrm{kg} / \mathrm{m} 2)$ was calculated. Ultrasound measurements recorded were the epidural depth $(\mathrm{ED}=$ distance from skin to the ligamentum flavum) and the dural sac width (DSW=distance between the inner surfaces of the dural sac). The ultrasound imaging was performed with the patient in the sitting position, at the L3/4 interspace, using the transverse approach, with a portable Titan Ultrasound System equipped with a 5.0-MHz curved array probe (Sonosite Canada Inc.). Data were analyzed by simple linear regression.

RESULTS: Patients' variables were as folows: maternal age $33 \pm 4.6$ yrs, height $164.5 \pm$ $6.4 \mathrm{~cm}$, weight $80.2 \pm 12.9 \mathrm{~kg}$, BMI $29.7 \pm 4.8$. Ultrasound measurements were: ED 4.68 $\pm 0.67 \mathrm{~cm}$, DSW $0.85 \pm 0.17 \mathrm{~cm}$. Univariate analysis of the ED showed significant correlation with patients'weight ( $\mathrm{r}=0.61$; adjust $\mathrm{r} 2=0.36, \mathrm{P}<0.001)$ and $\mathrm{BMI}(\mathrm{r}=0.59$; adjust $\mathrm{r} 2=0.34, \mathrm{P}<0.001$ ). No significant correlation was found between DSW and patient's anthropometric variables or between DSW and ED.

DISCUSSION: Our results are consistent with previous studies correlating ED with obstetric patients' anthropometric variables, either by means of epidural needle measurement $(2,3)$ or by ultrasound imaging (4). To our knowledge this is the first attempt to correlate the DSW with anthropometric variables. Unfortunately, no significant correlation could be established. It remains to be clarified whether the DSW can be used as a predictor of the final result of spinal anesthesia, similar to CSF volume REFERENCES: (1) Anesthesiology 1996; 84: 1341-1349; (2) Reg Anesth 1996: 21(5): 451-455; (3) Anesthesiology 102,Supp 1: A37; (4) Br J Anaesth 2001; 86: 798-804 
